\title{
Partial electron localization in a finite-size superlattice placed in an electric field
}

(C) K.R. Vlasov, M.A. Pyataev $₫$, A.V. Shorokhov

National Research Mordovia State University,

430005 Saransk, Russia

ฯ E-mail: pyataevma@gmail.com

Partial electron localization in a finite-size superlattice placed in an electric field is considered. The role of electric field in forming of quasilocalized states is investigated. A quantitative criterion for the degree of partial localization is suggested based on analysis of maximal probability density of finding an electron at a given point. It is found that with increase in the electric field the degree of localization does not increase monotonically. Furthermore, the localization is affected stronger by the amplitude of superlattice potential than by the electric field.

\section{Acknowledgements}

The work has been supported by the RFBR (grant No 17-02-00969). 